\title{
MicroRNA-548k upregulates a spliced variant of human CD2-associated protein by targeting its promoter
}

\author{
Nannan LiB, , Guoping Zhou ${ }^{\mathrm{A}}$ \\ Department of Pediatrics, First Affiliated Hospital, Nanjing Medical University, China \\ A - research concept and design; $\mathrm{B}$ - collection and/or assembly of data; $\mathrm{C}$ - data analysis and interpretation; \\ $D$ - writing the article; $E$ - critical revision of the article; $F$ - final approval of the article
}

Address for correspondence

Guo-Ping Zhou

E-mail: zhouguoping@jsph.org.cn

\section{Funding sources}

This work was funded by the National Natural Science Foundation of China (grants: №. 81170661, No. 31640048 to GPZ, No. 81300023 to RJ, and No. 81500013 to LLZ), the Provincial Natural Science Foundation of Jiangsu (grant No. BK20131020 to RJ), and the Nanjing Science and Technology Plan Project (grant No. 201503003 to GPZ)

Conflict of interest

None declared

Received on November 1, 2017 Reviewed on January 11,2018 Accepted on May 7, 2020

Published online on July 1,2020

\begin{abstract}
Background. MicroRNAs (miRNAa) regulate gene expression by repressing the translation or directing the degradation of mRNA in the cytoplasm. However, recent studies have shown that miRNAs act to regulate transcription at target gene promoters.
\end{abstract}

Objectives. To investigate whether miR-548k regulates CD2AP002 expression at the transcriptional level.

Material and methods. The CD2AP002 promoter region was cloned and its promoter activity was examined in HEK293 cells using luciferase assay. The CD2AP002 promoter region was characterized bioinformatically. The HEK293 cells were treated with a miR-548k mimic or inhibitor and the luciferase activity of the CD2AP002 promoter and the mRNA expression of CD2AP002 were assayed. A chromatin immunoprecipitation assay was performed with antibody against RNA polymerase ll at the CD2AP002 promoter region in HEK293 cells.

Results. The CD2AP002 promoter region contained 2 putative miR-548k binding sites, and mutational analysis revealed that both of those sites contributed to transcriptional activity. The miR-548k mimics increased CD2AP002 promoter activity and mRNA expression. In contrast, the miR-548k inhibitor significantly reduced CD2AP002 promoter activity and mRNA expression. Finally, the chromatin immunoprecipitation assay showed that the enrichment of RNA polymerase II at the CD2AP002 promoter region was enhanced in the HEK293 cells treated with the miR-548k mimic.

Conclusions. This is the first study to demonstrate that miR-548k upregulates CD2AP002 expression by targeting its promoter.

Key words: transcriptional regulation, gene promoters, CD2-associated protein, microRNA-548k, spliced variant

Cite as

Li N, Zhou G. MicroRNA-548k upregulates a spliced

variant of human $C D 2$-associated protein by targeting its promoter. Adv Clin Exp Med. 2020;29(6):677-682. doi:10.17219/acem/122168

DOI

10.17219/acem/122168

Copyright

Copyright by Author(s)

This is an article distributed under the terms of the

Creative Commons Attribution 3.0 Unported (CC BY 3.0)

(https://creativecommons.org/licenses/by/3.0/) 


\section{Introduction}

Primary focal segmental glomerulosclerosis (FSGS) is a destructive type of nephrotic syndrome and the main cause of primary nephropathy. ${ }^{1}$ Several gene mutations have been found in patients with primary FSGS, including the CD2-associated protein (CD2AP). The human CD2AP is an $80-\mathrm{kDa}$ protein that was originally cloned as a protein involved in $\mathrm{T}$ cell activation. ${ }^{2}$ In $\mathrm{T}$ cells, CD2AP binds the adhesion molecule CD2 and modulates the cytoskeletal polarity. CD2AP knockout mice exhibit high proteinuria at a young age followed by glomerulosclerosis, and they die from renal failure. The histological characteristics of the kidney lesions of CD2AP-deficient mice are similar to human FSGS. ${ }^{3}$

Alternative pre-messenger RNA (pre-mRNA) splicing plays an important role in the disease and its development. At least $74 \%$ of human multi-exon genes are alternatively spliced. ${ }^{4}$ Alternative splicing is a general mechanism for regulating gene expression and is associated with numerous diseases, such as thalassemia syndromes and dementia. ${ }^{5,6} \mathrm{CD} 2 \mathrm{AP}$ includes 5 spliced variants which may regulate the wild-type CD2AP. However, the mechanism underlying the regulation of the spliced variant of CD2AP remains unknown.

MicroRNAs (miRNAs) are a class of small non-coding RNAs that regulate gene expression by repressing translation or directing sequence-specific degradation of complementary mRNA in the cytoplasm. ${ }^{7}$ However, recent studies have shown that miRNAs act to regulate the stability of nuclear transcripts, silencing or activating transcription at the target gene promoters. ${ }^{8-12} \mathrm{miR}-548$ is a large and poorly conserved primate-specific miRNA gene family. There are 69 human miR-548 genes, located in almost all human chromosomes; this widespread distribution pattern indicates an evolutionary origin from transposable elements. ${ }^{13}$ However, the role of miR-548k remains elusive. In this study, we aimed to investigate whether miR-548k may regulate $\mathrm{CD} 2 \mathrm{AP} 002$ expression at the transcriptional level. Because CD2AP function is implicated in renal diseases such as FSGS, we selected the human embryonic kidney cell line HEK293 as the experimental model and employed different approaches to characterize the regulation of CD2AP002 transcription by miR-548K.

\section{Material and methods}

\section{Cell culture and transfection}

Human embryonic kidney HEK293 cells were purchased from the American Type Culture Collection (Manassas, USA), and maintained in Dulbecco's modified Eagle's medium (DMEM) supplemented with 10\% heat-inactivated fetal bovine serum (FBS), $100 \mathrm{U} / \mathrm{mL}$ of penicillin and $100 \mu \mathrm{g} / \mathrm{mL}$ of streptomycin in a humidified atmosphere of $5 \% \mathrm{CO}_{2}$ at $37^{\circ} \mathrm{C}$. Transfections were carried out in HEK293 cells using Lipofectamine 3000 (Invitrogen, Carlsbad, USA) according to the manufacturer's protocol.

\section{Plasmids}

A 2,827 bp of the 5'-flanking region of the human CD2AP002 gene was cloned using human genomic DNA extracted from the HEK293 cells as the template and primers synthesized by Invitrogen (the sequences were CGGGGTACCGAGATAACAAAAACAGATACCGAAG and GGAAGATCTTCTTTACCATTAAGTTCGCCCCTC, respectively, with the Kpn I and Bgl II restriction sites in bold and underlined). The polymerase chain reaction (PCR) was carried out using high-fidelity DNA polymerase. The PCR protocol was 30 cycles at $98^{\circ} \mathrm{C}$ for $10 \mathrm{~s}, 60^{\circ} \mathrm{C}$ for $15 \mathrm{~s}$ and $68^{\circ} \mathrm{C}$ for $3 \mathrm{~min}$. The PCR product was digested with Kpn I and Bgl II and ligated into a pGL3-basic vector to generate pGL3-CD2AP002 (named pGL3-2827). The clones were verified with sequencing. pGL3-2827 was scanned for sites complementary to known human miRNAs, using online RegRNA software (http:// regrna.mbc.nctu.edu.tw/html/prediction.html).

A deletion mutant of the plasmid containing the 5'-flanking region of CD2AP002 was generated with PCR using pGL3-2827 as the template and primers synthesized by Invitrogen (the sequences were CGGGGTACCTCTAAGTCCTGAAAACTTGGCACG and GGA AGATCTTCTTTACCATTAAGTTCGCCCCTC, respectively, with Kpn I and Bgl II restriction sites in bold and underlined). The plasmid was named pGL3-2200.

Site-directed mutagenesis of the putative miR-548k binding sites in the promoter region of CD2AP002 was performed with a Fast Mutagenesis System (Invitrogen). The sequence containing the $1^{\text {st }}$ miR-548k binding site was mutated from TACGGTATTGACTATAAGTATTTTA to the miR-548k mutation TACGGTATTGACTATAAATATTTTA, and the sequence containing the $2^{\text {nd }}$ miR-548k binding site was mutated from TTGTTCTTTTTTAAGTACTTT to the mutation TTGTTCTTTTTTAAGTAGTTT (mutations shown in bold). The mutations were confirmed with sequencing.

\section{Double-stranded miRNA and inhibitor}

The miR-548k mimic, the miR-548k inhibitor, the mismatched derivatives miR-548k-5MM, and the corresponding nonspecific control dsRNA (negative control) were synthesized with RiboBio (Guangzhou, China).

\section{Luciferase assay}

HEK293 cells were cultured for $24 \mathrm{~h}$ and then transfected with reporters and controls. The luciferase assay was performed $24 \mathrm{~h}$ after transfection using the dual-luciferase reporter gene assay kit (Promega; Madison, USA) according to the manufacturer's instructions. 


\section{Real-time PCR}

Total RNA, including miRNA, was extracted using Trizol reagent (Invitrogen). A total of $1 \mu \mathrm{g}$ of RNA was reverse-transcribed into cDNA with a PrimeScript RT Master Mix Perfect kit (Takara, Dalian, China). Realtime PCR (RT-PCR) was performed using SYBRR Premix Ex TaqTM (Takara, Dalian, China) and an ABI PRISM 7700 sequence detection system (PE Applied Biosystems, Wellesley, USA). The primers were synthesized by Invitrogen with sequences as follows: CD2AP002-F CTCCAAAGCCTGAACTGATAGC, CD2AP002-R AAATGAACTACTCCCTCCCAGA and GAPDH-F TGGTATCGTGGAAGGACTCATGAC, and GAPDH-R TGCCAGTGAGCTTCC CGTTCAGC. The reaction conditions were $95^{\circ} \mathrm{C}$ for $10 \mathrm{~min}$ ( 1 cycle), followed by 40 cycles of $95^{\circ} \mathrm{C}$ for $15 \mathrm{~s}, 60^{\circ} \mathrm{C}$ for $15 \mathrm{~s}$ and $70^{\circ} \mathrm{C}$ for $1 \mathrm{~min}$. GAPDH was used as the internal reference.

To detect the level of miR-548k, the miRNA was converted into cDNA with reverse transcription using a TaqMan MicroRNA reverse transcription kit (Applied Biosystems). To quantify miR-548k expression, RT-PCR was performed with the use of a TaqMan miRNA assay kit for hsa-miR-548k (Applied Biosystems). The primers were synthesized by Invitrogen with the following sequences: miR-548k Forward TCGGCGGAAAAGTACTTGCGGAT, Reverse ATCCAGTGCAGGGTCCGAGG and U6 Forward GGTCGGGCAGGAAAGAGGGC, Reverse GCTAATCTTCTCTGTATCGTTCC. The PCR protocol was $95^{\circ} \mathrm{C}$ for $5 \mathrm{~min}$ (1 cycle), followed by $40 \mathrm{cy}$ cles of $90^{\circ} \mathrm{C}$ for $15 \mathrm{~s}, 60^{\circ} \mathrm{C}$ for $15 \mathrm{~s}$ and $72^{\circ} \mathrm{C}$ for $1 \mathrm{~min}$. U6 served as an endogenous control to normalize miR-548k expression. The relative expression levels were calculated using the equation: Folds $=2^{-\Delta \Delta C t}$. Each sample was analyzed 3 times.

\section{Chromatin immunoprecipitation assay}

The HEK293 cells were transfected with miR-548k mimics or negative control miRNA with a scramble sequence (miR-NC). The cells were cross-linked with $1 \%$ formaldehyde for $10 \mathrm{~min}$ at room temperature before being stopped with glycine. Using a Magna/EZ-Magna chromatin immunoprecipitation assay (ChIP) Kit (Upstate Biotechnology, Lake Placid, USA), the cells were washed with cold PBS and harvested in lysis buffer supplemented with Protease Inhibitor Cocktail II (Upstate Biotechnology). Sonication of cross-linked chromatin was performed to obtain 200-1,000-bp chromatin fragments. Immunoprecipitation was performed using $5 \mu \mathrm{g}$ each of Anti-Acetyl Histone H3 (Upstate 06-599B; Upstate Biotechnology), Anti-IgG (Upstate PP64B; Upstate Biotechnology) and $10 \mu \mathrm{g}$ of Anti-RNA polymerase II (Santa Cruz sc-899X; Santa Cruz Biotechnology, Santa Cruz, USA) overnight at $4^{\circ} \mathrm{C}$ with rotation. In order to recover the antibody/antigen/DNA complexes, $20 \mu \mathrm{L}$ of Protein
A beads (Upstate Biotechnology) were added, and the mixture was washed 4 times. The eluted samples were treated with Proteinase $\mathrm{K}$ (Upstate Biotechnology) and incubated for $2 \mathrm{~h}$ at $62^{\circ} \mathrm{C}$ and $10 \mathrm{~min}$ at $95^{\circ} \mathrm{C}$ to reverse the crosslinks. DNA purification was performed using spin columns (Upstate Biotechnology). The purified DNA was amplified by promoter specific primers as follows: ChIP-F1 ATGTATCTCCTTGTAGTTGAATCTG and ChIP-R1 ACTTATTTAGCCTCTATCTTGTGTA (targeting the $1^{\text {st }}$ putative miR-548k binding sites on CD2AP002 promoter); ChIP-F2 GCACGTAATAAGTGCTCAG and ChIP-R2 AATAGAATGAGGTGGAAGA (targeting the $2^{\text {nd }}$ putative miR-548k binding sites on the CD2AP002 promoter). The input of each sample was used as an internal control. The PCR products were visualized with gel electrophoresis on $2 \%$ agarose gel, and confirmed with sequencing.

\section{Statistical analysis}

The data is presented as means \pm standard deviation (SD) of 3 or more independent results. Statistical significance was assessed using a two-tailed, unpaired Student's t-test. A p-value $<0.05$ was selected as the level denoting significant differences.

\section{Results}

\section{miR-548k upregulates CD2AP002 expression by binding to its promoter in HEK293 cells}

Scanning analysis of the CD2AP002 promoter showed that 2 sequences located at positions -2537 and -2041 relative to the transcription start site were highly complementary to miR-548k (Fig. 1A-C). To confirm that miR-548k upregulates CD2AP002 expression, we overexpressed miR-548k in HEK293 cells (Fig. 1D). Luciferase assay showed that the CD2AP002 promoter (pGL3-2827), which had 2 putative miR-548k binding sites, exhibited significantly higher luciferase activity in the HEK293 cells with an overexpression of miR-548K compared to the control cells (Fig. 1E). Furthermore, RT-PCR showed that CD2AP002 mRNA level was significantly higher in the HEK 293 cells with an overexpression of miR-548K compared to the control cells (Fig. 1F).

To determine whether the induction of CD2AP002 promoter activity was specific to the sequence of miR-548k, we performed deletion mutation and point mutation of 2 putative miR-548k binding sites. The luciferase assay revealed that both basic and miR-548k-stimulated luciferase activities were significantly reduced in miR-548k site-directed deletion mutation and twopoint mutations in the CD2AP002 promoter compared to the wild-type promoter (Fig. 2A). Furthermore, we synthesized a miR-548k mutant to create a mismatch with 


\section{A}

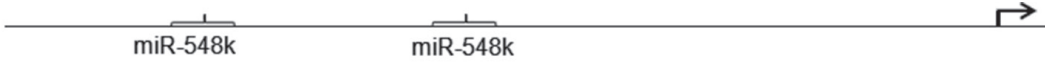

B 5'-ttgttactta acataCGGT, AT, 2537 GACTAT AAGT,ATTTT Taaa atttaaagat-3'

3'-ucGU'UUUA - - - GGCGUUCAUGAAAa-5'

$-2041$

5'- a accatcatat ttgttct TT TTT AAG TACT TTtttttttaaaat taacctcaac-3'

3'-ucguuuu AGGCGUUCAUGAAAa-5'

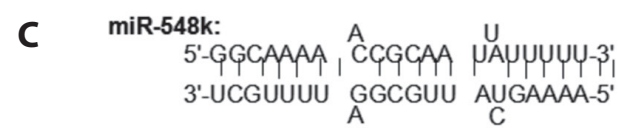

D

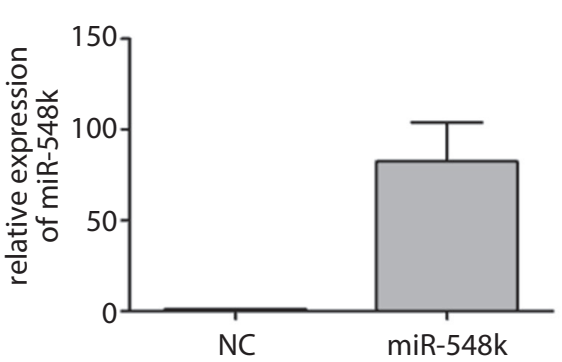

E

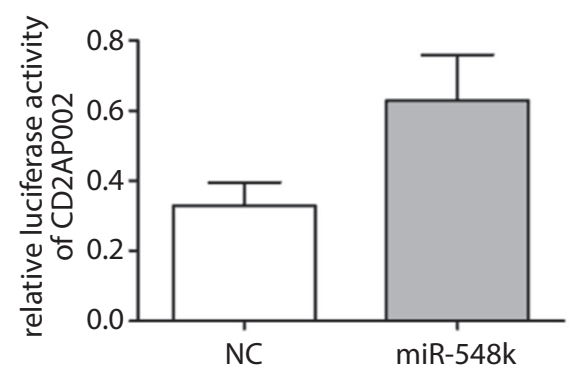

$\mathbf{F}$

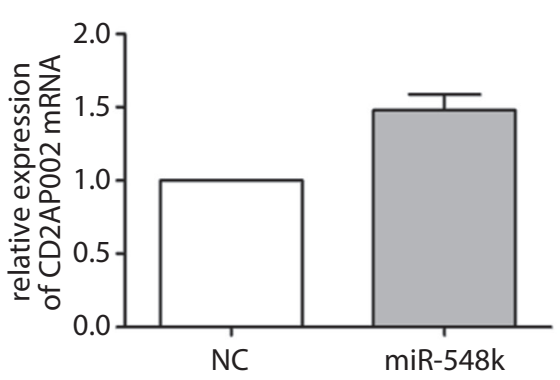

Fig. 1. miR-548k enhances gene expression by targeting the CD2AP002 promoter. A - schematic representation of the CD2AP002 promoter with 2 putative miR-548k target sites. B - sequences of miR-548k target sites located at nucleotides -2537 and -2041 relative to the transcription start site. C-sequence and structure of miR-548k. D - relative expression of miR-548k was determined with RT-PCR and normalized to U6. E - dual-luciferase reporter assay. F - relative expression of CD2AP002 was determined with real-time PCR and normalized to GAPDH. All data is presented as mean \pm SD from 3 independent trials

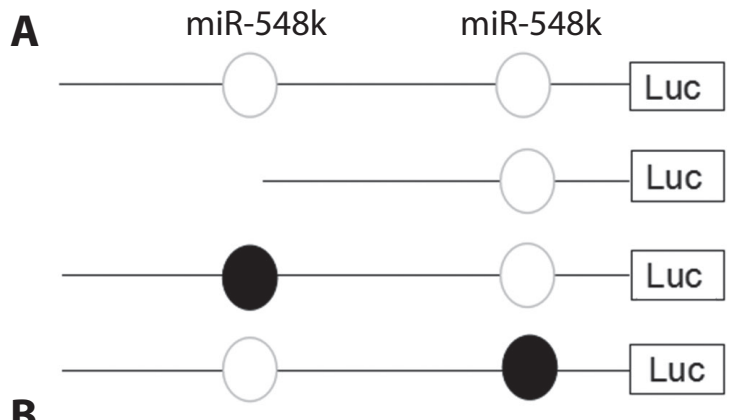

miR-548k:

A

5'-GGCAAAA CCGCAA UAUUUUU-3' || |||| |||||| || || ||

3'-UCGUUUU GGCGUU AUGAAAA-5'

A $\quad \mathrm{C}$

miR-548k-5MM:

$\mathrm{A}$

$\mathrm{U}$

5'-GGCAAAA CCGCAA UAUUUUU-3'

||||||| |||||| |||||||

3'-UCGUUUU GGCGUU AUCUGCA-5'

A

$\mathrm{C}$
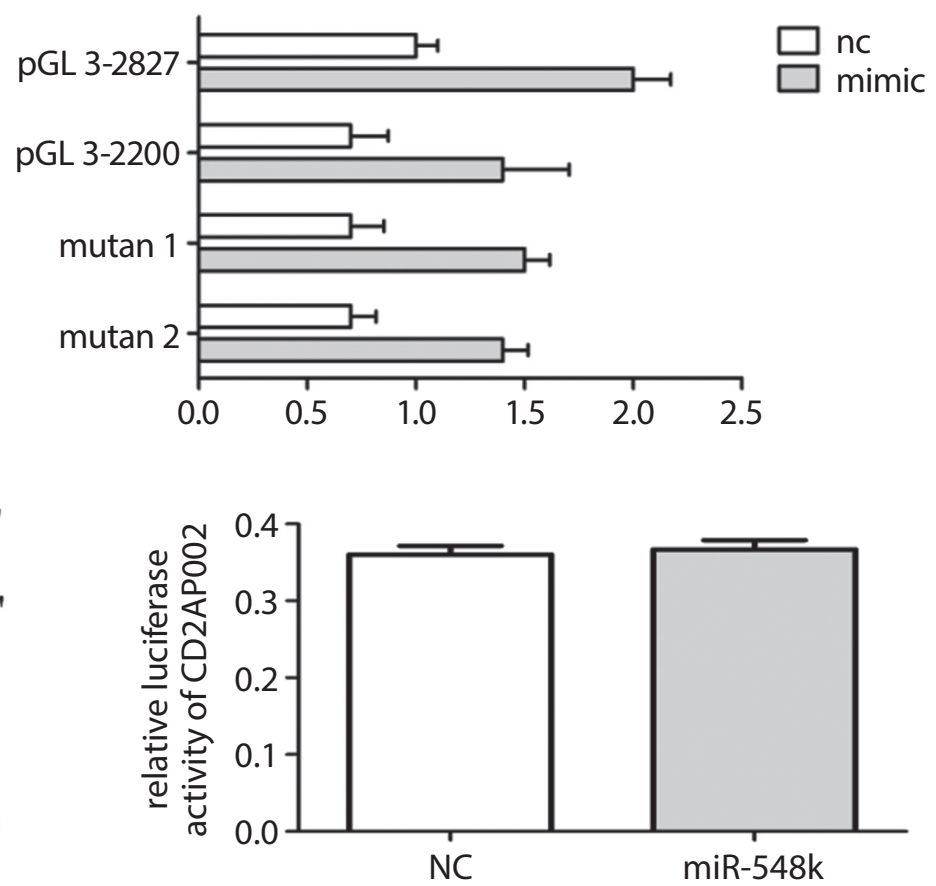

Fig. 2. Mutational analysis of human CD2AP002 promoter. A - left: schematic representation of the reporter constructs. The numbering is relative to the transcription start site; the binding sites for miR-548k are indicated with circles; point mutations are shown in bold. Right: luciferase activity of the reporter constructs in HEK293 cells transfected with $150 \mathrm{nM}$ of miR-548k mimic or miR-NC (negative control). B - left: mutation of 4 of the last base pairs in miR-548k resulted in miR-548k-5MM. The mutated bases are shown in bold. Right: luciferase activity of the reporter constructs in HEK293 cells transfected with $150 \mathrm{nM}$ of miR-548k-5MM or miR-NC (negative control). All data is presented as mean \pm SD from 3 independent trials 
A

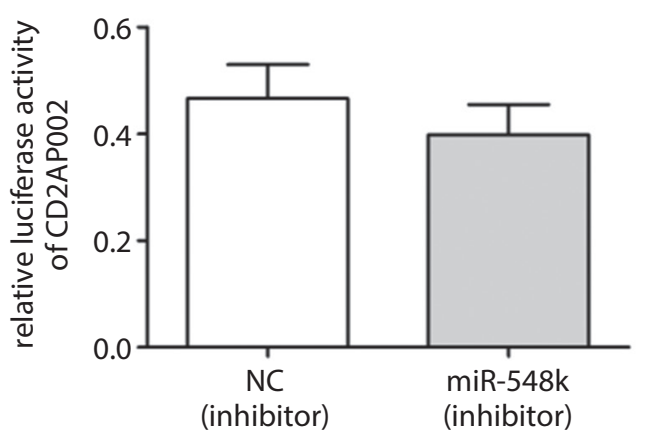

B

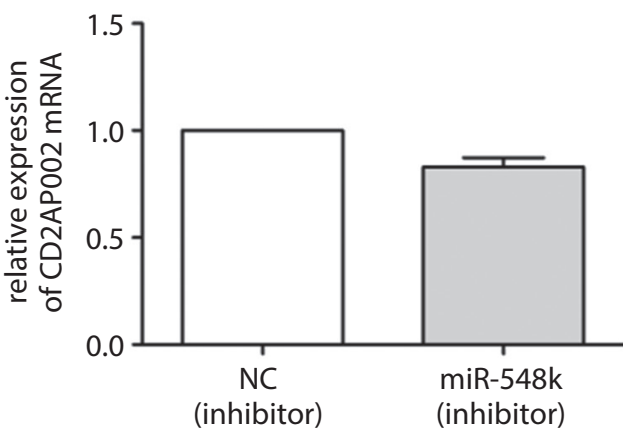

Fig. 3. miR-548k inhibitor represses the expression of CD2AP002. A - HEK293 cells were transfected with $200 \mathrm{nM}$ of miR-548k inhibitor or miR-NC (negative control) and reporter constructs. Dualluciferase reporter assay was performed $24 \mathrm{~h}$ later. B - relative expression of CD2AP002 was determined with RT-PCR and normalized to GAPDH in HEK293 cells transfected with $200 \mathrm{nM}$ of miR-548k inhibitor or miR-NC (negative control). All data is presented as mean \pm SD from 3 independent trials the target sites; the luciferase assay showed that this mutant miR-548k-5MM could not induce CD2AP002 promoter activity (Fig. 2B). Taken together, these findings indicate that miR-548k upregulates CD2AP002 expression by binding to 2 putative recognition sites in the promoter region of CD2AP002 in HEK293 cells.

\section{miR-548k inhibitor downregulates CD2AP002 expression in HEK293 cells}

To test whether the miR-548k inhibitor downregulated CD2AP002 expression, we transfected the HEK293 cells with a miR-548k inhibitor, which was designed specifically to bind to and sequester a mature miR-548k sequence, and its miR-NC as the control. Luciferase assay demonstrated that the miR-548k inhibitor reduced basic luciferase activity driven by the CD2AP002 promoter (Fig. 3A). Moreover, RT-PCR showed that the miR-548k inhibitor reduced the expression of CD2AP002 mRNA in HEK293 cells (Fig. 3B).

\section{Enrichment of miR-548k at the CD2AP002 promoter}

To confirm that miR-548k binds to the CD2AP002 promoter directly, we performed a ChIP assay. RNA polymerase II (pol II) holoenzyme is known to be recruited

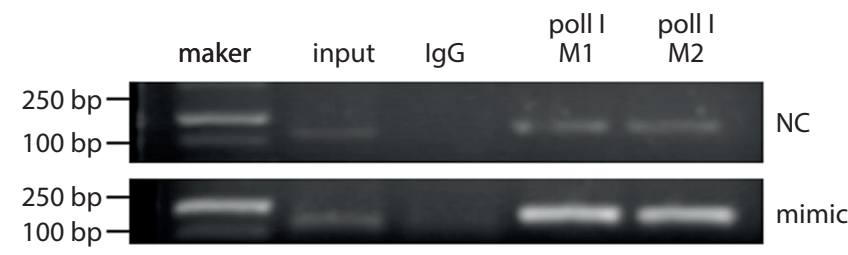

Fig. 4. Enrichment of pol II at CD2AP002 promoter by miR-548k. HEK293 cells were transfected with miR-NC (negative control) or the miR-548k mimic. At $24 \mathrm{~h}$ post-transfection, the cells were harvested and subjected to ChIP assay using a pol II-specific antibody or normal rabbit lgG (negative control) to immunoprecipitate transcriptionally active regions of DNA. Purified DNA was analyzed with RT-PCR using primers targeting 2 putative miR-548k binding sites on the CD2AP002 promoter. DNA pulled down in the absence of antibody (No Ab) served to identify background amplification. The input DNA was amplified as a loading control. Representative results from 3 independent trials with similar results are shown to the promoters of protein-coding genes in living cells. Thus, we used an antibody specific to pol II for ChIP and mapped 2 regions corresponding to the transcription start sites of CD2AP002. The PCR and sequencing results revealed that miR-548k was associated with the CD2AP002 promoter at an endogenous level in HEK293 cells. In the cells transfected with the miR-548k mimic, we found increased recruitment of miR-548k at CD2AP002 transcription start sites (Fig. 4). These results suggest that miR-548k is recruited to the CD2AP002 promoter to activate the transcription of CD2AP002.

\section{Discussion}

Alternative splicing is a key mechanism for expanding transcript and protein diversity of mammalian genes. CD2AP is a multi-domain scaffolding protein that has a critical role in renal function. Our previous studies have shown that the CD2AP promoter in human renal tubular cells could be regulated by CREB, Sp1 and E2F1. ${ }^{14}$ However, little is known about the regulation of alternative splicing of CD2AP. In this study, we focused on a splicing variant CD2AP002 and characterized its promoter in HEK293 cells.

Recent studies have indicated the role of non-coding RNAs in diseases. ${ }^{15}$ For instance, transgenic expression of miR-193a in mice rapidly induced FSGS with extensive podocyte foot process effacement. ${ }^{16}$ The concentrations of serum miR-30a-5p, miR-151-3p, miR-150, miR-191, and miR-19b were significantly higher in nephritic syndrome (NS) children than in controls. ${ }^{17}$ In addition, the expression of miR-141, miR-205 and miR-192 was correlated with disease severity and progression in patients with IgA nephropathy. ${ }^{18}$

Interestingly, miRNA-induced transcriptional regulation has been reported in mammalian systems. miR-373 could upregulate E-cadherin and CSDC2, both of which contained complementary sequences to miR-373 in their promoters. ${ }^{19}$ miR-320 could recruit AGO1 and other factors to silence POLR3D expression. ${ }^{20}$ Moreover, Younger et al. found that miRNAs could inhibit the expression 
of progesterone receptor (PR) by targeting its promoter. ${ }^{21}$ Recently, our group reported that miR-939 downregulated CD2AP expression by targeting its promoter in HEK-293T cells. ${ }^{22}$ However, in this study, we found that miR-548k could activate CD2AP002 expression at the transcriptional level and we identified 2 putative binding sites for miR-548k in the promoter region of CD2AP002. The sequence specificity was confirmed using deletion and point mutation assay. A ChIP assay further demonstrated that miR-548k activated transcription by direct binding to the promoter of CD2AP002.

However, the detailed mechanism by which miRNAs modulate transcriptional regulation by targeting promoters remains unclear. In the cytoplasm, the function of miRNAs is well known to direct an Argonaute-containing complex to target and repress or degrade mRNAs. More recently, a regulatory role for miRNAs and Argonaute has been reported in the nucleus, where miRNAs guide Argonaute to target gene promoters and directly regulate transcription. We assume that miR-548k could recruit Argonaute to a CD2AP002 promoter, overlapping the binding sites of some transcription factors, which therefore come into contact with transcription factors and RNA polymerase II in a positive manner. Further studies are required to test this possibility.

\section{Conclusions}

Our findings demonstrate for the first time that miR-548k upregulates CD2AP002 expression by targeting specific sites in its promoter region. Our study may provide new insight into the role of miRNAs in gene expression regulation and disease progression.

\section{References}

1. Dragovic D, Rosenstock JL, Wahl SJ, Panagopoulos G, DeVita MV Michelis MF. Increasing incidence of focal segmental glomerulosclerosis and an examination of demographic patterns. Clin Nephrol. 2005;63(1):1-7.

2. Wolf G, Stahl RA. CD2-associated protein and glomerular disease. Lancet. 2003;362(9397):1746-1748.

3. Shih NY, Li J, Karpitskii V, et al. Congenital nephrotic syndrome in mice lacking CD2-associated protein. Science. 1999;286(5438):312-315.
4. Johnson JM, Castle J, Garrett-Engele P, et al. Genome-wide survey of human alternative pre-mRNA splicing with exon junction microarrays. Science. 2003;302(5653):2141-2144.

5. Radmilovic M, Zukic B, Stankovic B, et al. Thalassemia syndromes in Serbia: An update. Hemoglobin. 2010;34(5):477-485.

6. Andreadis A. Tau gene alternative splicing: Expression patterns, regulation and modulation of function in normal brain and neurodegenerative diseases. Biochim Biophys Acta. 2005;1739(2-3):91-103.

7. Bartel DP. MicroRNAs: Genomics, biogenesis, mechanism, and function. Cell. 2004;116(2):281-297.

8. Kalantari R, Chiang CM, Corey DR. Regulation of mammalian transcription and splicing by nuclear RNAi. Nucleic Acids Res. 2016;44(2): 524-537.

9. Paugh SW, Coss DR, Bao J, et al. MicroRNAs form triplexes with double stranded DNA at sequence-specific binding sites: An eukaryotic mechanism via which microRNAs could directly alter gene expression. PLoS Comput Biol. 2016;12(2):e1004744.

10. Suzuki K, Ahlenstiel C, Marks K, Kelleher AD. Promoter targeting RNAs: Unexpected contributors to the control of HIV-1 transcription. Mol Ther Nucleic Acids. 2015;4(1):e222.

11. Cho S, Park JS, Kang YK. AGO2 and SETDB1 cooperate in promotertargeted transcriptional silencing of the androgen receptor gene. Nucleic Acids Res. 2014;42(22):13545-13556.

12. Benhamed M, Herbig U, Ye T, Dejean A, Bischof O. Senescence is an endogenous trigger for microRNA-directed transcriptional gene silencing in human cells. Nat Cell Biol. 2012;14(3):266-275.

13. Liang T, Guo L, Liu C. Genome-wide analysis of mir-548 gene family reveals evolutionary and functional implications.J Biomed Biotechnol. 2012;2012:679563.

14. Zou L, Xu HG, Ren W, Jin R, Wang Y, Zhou GP. Transcriptional activation of the human CD2AP promoter by E2F1. PLoS One. 2012;7(8):e42774.

15. Ni X, Liao Y, Li L, Zhang X, Wu Z. Therapeutic role of long non-coding RNA TCONS_00019174 in depressive disorders is dependent on Wnt/ $\beta$-catenin signaling pathway. J Integr Neurosci. 2018;17(2):125-132. doi:10.3233/JIN-170052

16. Gebeshuber CA, Kornauth C, Dong L, et al. Focal segmental glomerulosclerosis is induced by microRNA-193a and its downregulation of WT1. Nat Med. 2013;19(4):481-487.

17. Luo $Y$, Wang $C$, Chen $X$, et al. Increased serum and urinary microRNAs in children with idiopathic nephrotic syndrome. Clin Chem. 2013;59(4):658-666.

18. Wang G, Kwan BC, Lai FM, et al. Intrarenal expression of microRNAs in patients with IgA nephropathy. Lab Invest. 2010;90(1):98-103.

19. Place RF, Li LC, Pookot D, Noonan EJ, Dahiya R. MicroRNA-373 induces expression of genes with complementary promoter sequences. Proc Natl Acad Sci U S A. 2008;105(5):1608-1613.

20. Kim DH, Saetrom P, Snøve O Jr, Rossi JJ. MicroRNA-directed transcriptional gene silencing in mammalian cells. Proc Natl Acad Sci U S A. 2008;105(42):16230-16235.

21. Younger ST, Corey DR. Transcriptional gene silencing in mammalian cells by miRNA mimics that target gene promoters. Nucleic Acids Res. 2011;39(13):5682-5691.

22. Huang YP, Qiu LZ, Zhou GP. MicroRNA-939 downregulates CD2-associated protein by targeting promoter in HEK-293T cells. Ren Fail. 2016; 38(4):508-513. 